Macedonian Pharmaceutical Bulletin, 66 (Suppl 1) 173 - 174 (2020)

Online ISSN 1857 - 8969

UDC: 616.15:616.314-089.23

DOI: 10.33320/maced.pharm.bull.2020.66.03.086

Short communication

\title{
Monitoring the changes in ALP, AST and LDH activity during short-term orthodontic treatment using multivariate algorithms for chemometric data analysis
}

\author{
Liljana Anastasova ${ }^{1}$, Angela Tasevska ${ }^{2}$, Natasa Toseska Spasova $^{2}$, Mirjana Popovska $^{3}$, \\ Rumenka Petkovska ${ }^{1}$ \\ ${ }^{1}$ Institute of Applied Chemistry and Pharmaceutical Analysis, Faculty of Pharmacy, \\ Ss. Cyril and Methodius University, 1000 Skopje, Republic of North Macedonia \\ ${ }^{2}$ Department of Orthodontics, Faculty of Dentistry, Ss. Cyril and Methodius University, \\ 1000 Skopje, Republic of North Macedonia \\ ${ }^{3}$ Department of Oral Pathology and Periodontology, Faculty of Dentistry, Ss. Cyril and Methodius University, \\ 1000 Skopje, Republic of North Macedonia
}

\section{Introduction}

Chemometrics is a science of multidisciplinary nature which has a widespread application in different fields. In medicine and pharmacy, chemometric algorithms for multivariate data analysis, among other applications, are used to monitor patient's health as well the effects of the medical treatments (Mocak, 2012). Short-term orthodontic treatment consists of application of orthodontic force in order to produce tooth movement. Monitoring the activity of certain biomarkers in gingival crevicular fluid (GCF) could be a clinically useful and noninvasive procedure to assess the changes in periodontal tissues after orthodontic force application (d'Apuzzo et al., 2013; d'Apuzzo et al., 2017). Several enzymes in GCF, such as alkaline phosphatase (ALP), lactate dehydrogenase (LDH) and aspartate aminotransferase (AST) have been assessed during orthodontic treatment and could be used for monitoring tissue changes during treatment (d'Apuzzo et al., 2013; Perinetti et al., 2012). The aim of our study was to examine changes in the ALP, AST and LDH activity in GCF during the short term orthodontic treatment using chemometric algorithms for multivariate data analysis.

\section{Materials and methods}

The study protocol was approved by the Ethics Committee at the Faculty of Dentistry, Skopje. Twenty orthodontic patients aged from 13-28 were enrolled in the study. Informed consent was obtained from the patients after providing information about the study. Fixed orthodontic appliance, Roth 22 Dentaurum was used. GCF samples were collected from each patient before application of orthodontic force (t0) and after $7(\mathrm{t} 1), 14(\mathrm{t} 2)$ and 21 days (t3) of the treatment, from the mesial and distal side of the maxillary test canine. Four paper strips were inserted in the gingival crevice and left in place for $30 \mathrm{~s}$ (Koss et al., 2009). Afterwards, they were transferred in eppendorf tubes containing $250 \mu \mathrm{L}$ phosphate buffer saline (PBS, $\mathrm{pH}=7.4$ ) and stored at $-20{ }^{\circ} \mathrm{C}$ until analysis. ALP, LDH and AST activity in GCF samples was determined using commercially available kits, according to manufacturer's instructions. Chemometric algorithms for multivariate data analysis, principal component

\footnotetext{
*lbogdanovska@ff.ukim.edu.mk
} 
analysis (PCA) and the orthogonal projections to latent structures discriminat analysis (OPLS-DA) were applied using SIMCA 14.1 software (Umetrics, Umea, Sweden). The obtained data from ALP, AST and LDH activity during short term orthodontic treatment was divided into mesial data set and distal data set and pareto-scaled for multivariate data analysis.

\section{Results and discussion}

PCA was applied on the two datasets to investigate global data variability. Several outliers were detected for each data set and removed from further analysis. The OPLS-DA algorithm provides comprehensive description on the discrimination between classes of samples, especially in cases where subtle differences among classes are present (Bylesjo et al., 2006). This algorithm separates the systematic variation in the matrix X (ALP, AST and LDH activity in GCF in the selected time intervals) in two parts, one linearly related (variation of interest) to the matrix $\mathrm{Y}$ (the classification variables) and one orthogonally related (so-called orthogonal variation or structured noise) to the matrix $\mathrm{Y}$. OPLS-DA analysis was applied to investigate differences for each time interval and t0. The data for the biomarker activity at $\mathrm{t} 0$ and $\mathrm{t} 1$, for both distal and mesial data sets were overlapped. This finding might be due to time needed the "stressed" tissue to respond to mechanical stimuli. The score plots of OPLS-DA models comparing biomarker activity at $\mathrm{t} 0$ and $\mathrm{t} 2$, for both mesial and distal data sets resulted in clear separation of two groups. The cross validation parameters for OPLS-DA model for the mesial data set were $R^{2} X$ (cum) $=1, R^{2} Y($ cum $)=$ 0.579 and $\mathrm{Q}^{2} \mathrm{X}$ (cum) $=0.513$, indicating a good model. Based on the VIP statistics from the crossvalidated OPLS - DA model, statistically significant variables responsible for separation between the two treatment points (VIP value $>0.8$ ) were extracted. The most important variables responsible for separation in the mesial data set were ALP and AST activity. There was also clear separation between groups for the distal data set containing biomarker activity at $\mathrm{t} 0$ and $\mathrm{t} 2$, with the following model characteristics: $\mathrm{R}^{2} \mathrm{X}($ cum $)=1, \mathrm{R}^{2} \mathrm{Y}($ cum $)=0.404$ and $\mathrm{Q}^{2} \mathrm{X}(\mathrm{cum})=0.419$. According to the VIP criterion, ALP and AST activity in GCF were the most important variables for class separation. The comparison of biomarker activity at $\mathrm{t} 0$ and $\mathrm{t} 3$ for the mesial data set resulted in two distinct clusters of data and satisfactory model characteristics: $\mathrm{R}^{2} \mathrm{X}($ cum $)=0.803, \quad \mathrm{R}^{2} \mathrm{Y}(\mathrm{cum})=0.457$ and $\mathrm{Q}^{2} \mathrm{X}(\mathrm{cum})=0.415$. The most important biomarkers responsible for class separation were the ALP activity and AST activity. The score plot of the OPLS-DA model for the last time point of the distal data set also gave two groups and good model statistics: $\mathrm{R}^{2} \mathrm{X}(\mathrm{cum})=1, \mathrm{R}^{2} \mathrm{Y}(\mathrm{cum})=0.438$ and $\mathrm{Q}^{2} \mathrm{X}(\mathrm{cum})=0.401$. However, compared to the mesial data set, the most important variables responsible for class separation were ALP activity and LDH activity, whereas AST activity had no influence on class separation.

\section{Conclusion}

Multivariate chemometric data analysis was applied as an approach to evaluate changes in ALP, AST and LDH activity during short-term orthodontic treatment. This approach may be used as means to assess treatment progress or to avoid adverse effects of treatment on the periodontium.

\section{References}

Bylesjo, M., Rantalainen, M., Cloarec, O., Nicholson, J., Holmes, E., Trygg, J., 2006. OPLS discriminat analysis: combining the strengths of PLS-DA and SIMCA classification. J. Chemom. 20, 341-351.

d'Apuzzo, F., Cappabianca, S., Ciavarella, D., Monsurro, A., Silvestrini-Biavati, A., Perillo, L., 2013. Biomarkers of periodontal tissue remodeling during orthodontic tooth movement in mice and men: overview and clinical relevance. Sci. World J. ID 105873. Available at: https://doi.org/10.1155/2013/105873.

d'Apuzzo, F., Perillo, L., Delfino, I., Portaccio M., Lepore, M., Camerlingo, C., 2017. Monitoring early phases of orthodontic treatment by means of Raman spectroscopies. J. Biomed. Opt. 22(11), 1-10.

Koss, M., Castro, S., Lopez, M., 2009. Enzymatic profile of gingival crevicular fluid in association with periodontal status. Lab. Med. 40, 277- 280.

Mocak, J., 2012. Chemometrics in medicine and pharmacy. Nova Biotechnol. Chim. 11, 11- 25.

Perinetti, G., Di Leonardo, B., Lenarda, R., Contardo, L., 2012. Repeatability of gingival crevicular fluid collection and quantification, as determined through its alkaline phosphatase activity: implications for diagnostic use. J. Periodont. Res. 48, 98-104. 